\title{
Domain Identification for a Nonlinear Elliptic Equation
}

\author{
D. D. Trong
}

\begin{abstract}
It is proposed to identify the domain $\Omega \subset \mathbb{R}^{n}$ of a nonlinear elliptic equation subject to given Cauchy data on part of the known outer boundary $\Gamma$ and to the zero condition on the unknown inner boundary $\gamma$. It is proved that under the assumption $\dot{\bar{\Omega}}=\Omega$, the domain $\Omega$ is uniquely determined.
\end{abstract}

Keywords: Domain identification, nonlinear elliptic equations, zero Dirichlet condition AMS subject classiflcation: $35 \mathrm{R} 30,35 \mathrm{~J} 60$

Let $\Omega \subset \mathbb{R}^{n}$ be a bounded domain limited by an outer boundary $\Gamma$ and an inner boundary $\gamma$, where $\Gamma$ is known, but $\gamma$ is unknown. Let

$$
F: \mathbb{R}^{n} \times \mathbb{R} \times \mathbb{R}^{n} \times \mathbb{R}^{n^{2}} \longrightarrow \mathbb{R}
$$

be a continuously differentiable function. We consider the nonlinear partial differential equation

$$
F\left(x, u, D u, D^{2} u\right)=0 \quad(x \in \Omega),
$$

where $u=u(x), D u=\left(\frac{\partial u}{\partial x_{i}}\right)_{1 \leq i \leq n}$ and $D^{2} u=\left(\frac{\partial^{2} u}{\partial x_{i} \partial x_{j}}\right)_{1 \leq i, j \leq n}$, subject to the boundary conditions

$$
\left.u\right|_{\Gamma_{0}}=f,\left.\quad \frac{\partial u}{\partial n}\right|_{\Gamma_{0}}=g,\left.\quad u\right|_{\gamma}=0
$$

where $\Gamma_{0}$ is an open subset of $\Gamma$.

In the present paper, we consider domains $\Omega \subset \mathbb{R}^{n}$ satisfying

$$
\stackrel{\circ}{\Omega}=\Omega
$$

where $\AA$ is the interior of the set A. Our problem is to determine a pair $(\Omega, u)$ satisfying (1) - (2). The case that $u$ is a harmonic function and the interior boundary $\gamma$ is a starshaped Jordan curve was considered in [1]. The present paper extends [1] in two ways. First, our equation is a fully nonlinear elliptic one (satisfying the maximum principle)

D. D. Trong: Hochiminh City Nat. Univ., Dept. Math. \& Comp. Sci.,-227 Nguyen Van Cu str., Q5 Hochiminh City, Vietnam 
curves that are the boundaries of simply connected subdomains with mutually disjoint closures. For physical applications of the problem, the reader is referred to, e.g., [1] and the references therein.

We assume that

$$
d(\Gamma, \gamma)>0
$$

where $d(A, B)$ is the distance between two subsets $A, B \subset \mathbb{R}^{n}$. We say that equation (1) is elliptic if

$$
\sum_{i, j=1}^{n} \frac{\partial F}{\partial q_{i j}}(x, u, p, q) \xi_{i} \xi_{j} \geq C|\xi|^{2}
$$

with a constant where $C>0$, for all $\xi=\left(\xi_{1}, . ., \xi_{n}\right) \in \mathbb{R}^{n},|\xi|^{2}=\xi_{1}^{2}+\ldots+\xi_{n}^{2}, x \in \Omega$, $(u, p, q) \in \mathbb{R} \times \mathbb{R}^{n} \times \mathbb{R}^{n^{2}}$ and $q=\left(q_{i j}\right)_{1 \leq i, j \leq n}$. We also assume that

$$
\begin{aligned}
& \frac{\partial F}{\partial u}(x, u, p, q) \leq 0 \quad \text { for all }(x, u, p, q) \in \mathbb{R}^{n} \times \mathbb{R} \times \mathbb{R}^{n} \times \mathbb{R}^{n^{2}} \\
& F(x, 0,0,0)=0 \quad \text { for all } x \in \mathbb{R} \text {. }
\end{aligned}
$$

We note here that if $F(x, u, p, q)=q_{11}+q_{22}+\ldots+q_{n n}$, then $F\left(x, u, D u, D^{2} u\right)=\Delta u$.

We have the following result.

Theorem. If $f \not \equiv 0$ or if $g \not \equiv 0$ on $\Gamma_{0}$, then there exists at most one pair $(\Omega, u)$ with $u \in C^{2}(\Omega) \cap C^{1}\left(\Omega \cup \Gamma_{0}\right) \cap C(\bar{\Omega})$ satisfying (1) - (2) provided (3) - (7) hold.

Proof. Suppose $\left(\Omega^{i}, u^{i}\right)$ satisfy (1) - (2) with

$$
u^{i} \in C^{2}\left(\Omega^{i}\right) \cap C^{1}\left(\Omega^{i} \cup \Gamma_{0}\right) \cap C\left(\overline{\Omega^{i}}\right)
$$

and let $\gamma^{i}$ be the inner boundaries of $\Omega^{i} \quad(i=1,2)$. Put

$$
\omega=\left\{x \in \Omega^{1}: d(x, \Gamma)<\min \left\{d\left(\Gamma, \gamma^{1}\right), d\left(\Gamma, \gamma^{2}\right)\right\}\right\}
$$

By (4), $\omega$ is connected and $\omega \neq \emptyset$. By the properties of $\Omega^{i}$ we have $\omega \subset \Omega^{1} \cap \Omega^{2}$. Let $W$ be the connected component of $\Omega^{1} \cap \Omega^{2}$ such that $\omega \subset W$. We shall prove that

$$
u^{1}=u^{2} \quad \text { on } W \subset \Omega^{1} \cap \Omega^{2} .
$$

Putting $v=u^{2}-u^{1}$ and

$$
\begin{aligned}
& u^{0}(x, t)=u^{1}(x)+t\left(u^{2}(x)-u^{1}(x)\right) \\
& p^{0}(x, t)=D u^{1}(x)+t\left(D u^{2}(x)-D u^{1}(x)\right) \\
& q^{0}(x, t)=D^{2} u^{1}(x)+t\left(D^{2} u^{2}(x)-D^{2} u^{1}(x)\right)
\end{aligned}
$$

we have

$$
g(x, 1)-g(x, 0)=\int_{0}^{1} \frac{\partial g}{\partial t}(x, t) d t
$$


where

$$
g(x, t)=F\left(x, u^{0}(x, t), p^{0}(x, t), q^{0}(x, t)\right) .
$$

This gives

$$
\sum_{i, j=1}^{n} a_{i j}(x) \frac{\partial^{2} v}{\partial x_{i} \partial x_{j}}+\sum_{i=1}^{n} b_{i}(x) \frac{\partial v}{\partial x_{i}}+c(x) v=0
$$

where

$$
\left.\begin{array}{rl}
a_{i j}(x) & =\int_{0}^{1} \frac{\partial F}{\partial q_{i j}}\left(x, u^{0}, p^{0}, q^{0}\right) d t \\
b_{i}(x) & =\int_{0}^{1} \frac{\partial F}{\partial p_{i}}\left(x, u^{0}, p^{0}, q^{0}\right) d t \\
c(x) & =\int_{0}^{1} \frac{\partial F}{\partial u}\left(x, u^{0}, p^{0}, q^{0}\right) d t
\end{array}\right\}
$$

Since $\Gamma \subset \partial W$, we get from (2)

$$
\left.v\right|_{\Gamma_{0}}=0 \quad \text { and }\left.\quad \frac{\partial v}{\partial n}\right|_{\Gamma_{0}}=0
$$

In view of $(10),(12)$ and (6), we can use the uniqueness theorem for the Cauchy problem for elliptic equations (see, e.g., [3]) to get $v=0$ on $W$, i.e. (9) holds. To continue with the proof, we suppose by contradiction that $\Omega^{1} \neq \Omega^{2}$. Without loss of generality, we can assume that $\Omega^{1} \backslash \overline{\Omega^{2}} \neq \emptyset$ (in fact, if $\Omega^{1} \backslash \overline{\Omega^{2}}=\Omega^{2} \backslash \overline{\Omega^{1}}=\emptyset$, then $\overline{\Omega^{1}}=\overline{\Omega^{2}}$, and by (3), $\Omega^{1}=\dot{\circ} \overline{\Omega^{1}}=\dot{\circ} \bar{\Omega}^{2}=\Omega^{2}$ ). Since $\Omega^{1} \backslash \overline{\Omega^{2}} \subset \Omega^{1} \backslash \bar{W}$ we have

$$
\Omega^{1} \backslash \bar{W} \neq \emptyset \text {. }
$$

Let $U$ be a connected component of $\Omega^{1} \backslash \bar{W}$. In view of (8), we have

$$
U \subset\left\{x \in \Omega^{1}: d(x, \Gamma) \geq \min \left\{d\left(\Gamma, \gamma^{1}\right), d\left(\Gamma, \gamma^{2}\right)\right\}\right\}
$$

Hence

$$
\partial U \cap \Gamma=\emptyset
$$

Note that

$$
\partial U \subset \partial\left(\Omega^{1} \backslash \bar{W}\right)=\partial\left(\Omega^{1} \cap\left(\mathbb{R}^{n} \backslash \bar{W}\right)\right) \subset \partial \Omega^{1} \cup \partial W .
$$

We can combine the above inclusion with (15) to get

$$
\partial U \subset \gamma^{1} \cup \partial W
$$

We claim that

$$
\left.u^{1}\right|_{\partial u}=0
$$


In fact, for $x \in \partial U$, there are two cases:

(a) $x \in \gamma^{1}$. In this case, (2) gives

$$
u^{1}(x)=0
$$

(b) $x \notin \gamma^{1}$. By (16), $x \in \partial W \backslash \gamma^{1}$. But $\partial W \subset \partial\left(\Omega^{1} \cap \Omega^{2}\right) \subset \gamma^{1} \cup \gamma^{2}$. Hence $x \in \gamma^{2}$. We prove that $x \in \Omega^{1}$. Indeed, $x \in \partial U \subset \overline{\Omega^{1}}$. From (15), we get

$$
x \in \overline{\Omega^{1}} \backslash\left(\Gamma \cup \gamma^{1}\right)=\overline{\Omega^{1}} \backslash \partial \Omega^{1}=\Omega^{1} .
$$

In this case, one has in view of (9)

$$
u^{1}(x)=u^{2}(x)=0 .
$$

In either case, from (18) and (19) we have that (17) holds.

Similarly as for (10), we get in view of (7)

$$
\sum_{i, j=1}^{n} a_{i j}^{1}(x) \frac{\partial^{2} u^{1}}{\partial x_{i} \partial x_{j}}+\sum_{i=1}^{n} b_{i}^{1}(x) \frac{\partial u^{1}}{\partial x_{i}}+c^{1}(x) u^{1}=0
$$

for $x \in \Omega^{1}$, where $a_{i j}^{1}, b_{i}^{1}, c^{1}$ have the same forms as in (11). We do not write out their explicit forms but only note that $(20)$ is an elliptic equation with

$$
c^{1}(x) \leq 0
$$

Using the maximum principle for elliptic equations (see [2: Chapter 2/p. 53]), we get in view of (17) and (21)

$$
u^{1}(x)=0 \quad \text { on } U \text {. }
$$

Now, using the uniqueness result for elliptic continuation [3], we get from $(22) u^{1}(x)=0$ on $\Omega^{1}$. Hence

$$
\left.u^{1}\right|_{\Gamma_{0}}=\left.\frac{\partial u^{1}}{\partial n}\right|_{\Gamma_{0}}=0
$$

This is a contradiction and the proof of the theorem is completed

\section{References}

[1] Ang, D. D. and L. K. Vy: Domain identification for harmonic functions. Acta Appl. Math. 38 (1995), 217 - 238.

[2] Friedman, A.: Partial Diferential Equations of Parabolic Type. Englewood Cliffs (N.J.): Prentice Hall 1964.

[3] Pederson, R. N.: On the unique continuation theorem for certain second and fourth order elliptic equations. Comm. Pure Appl. Math. 11 (1958), $67-80$. 